\title{
COMPOUND POISSON PROCESS WITH A POISSON SUBORDINATOR
}

\author{
ANTONIO DI CRESCENZO *** AND \\ BARBARA MARTINUCCI, ${ }^{* * * *}$ Università degli Studi di Salerno \\ SHELEMYAHU ZACKS, ${ }^{* * * *}$ Binghamton University
}

\begin{abstract}
A compound Poisson process whose randomized time is an independent Poisson process is called a compound Poisson process with Poisson subordinator. We provide its probability distribution, which is expressed in terms of the Bell polynomials, and investigate in detail both the special cases in which the compound Poisson process has exponential jumps and normal jumps. Then for the iterated Poisson process we discuss some properties and provide convergence results to a Poisson process. The first-crossing time problem for the iterated Poisson process is finally tackled in the cases of (i) a decreasing and constant boundary, where we provide some closed-form results, and (ii) a linearly increasing boundary, where we propose an iterative procedure to compute the first-crossing time density and survival functions.
\end{abstract}

Keywords: Bell polynomials; first-crossing time; iterated process; linear boundary; mean sojourn time; Poisson process

2010 Mathematics Subject Classification: Primary 60J27; 60G40

\section{Introduction}

Stochastic processes evaluated at random times are receiving increasing attention in various applied fields. There are many examples of processes with random times that could be modeled in this manner:

(i) in reliability theory, the life span of items subject to accelerated conditions,

(ii) in econometrics, the composition of the price of a security and the effective economic time,

(iii) in queueing theory, the number of customers joining the system during specific service periods,

(iv) in statistics, for the random sampling of stochastic processes.

One of the first papers in this field is Lee and Whitmore [10], who studied general properties of processes directed by randomized times. In the literature special attention is given to the case of a Poisson process with randomized time. Another earlier example of a stochastic process with

\footnotetext{
Received 17 December 2013; revision received 23 June 2014.

* Postal address: Dipartimento di Matematica, Università degli Studi di Salerno, Via Giovanni Paolo II, n. 132, 84084 Fisciano (SA), Italy.

** Email address: adicrescenzo@unisa.it

*** Email address: bmartinucci@unisa.it

**** Postal address: Department of Mathematical Sciences, Binghamton University, Binghamton, NY 13902-6000, USA. Email address: shelly@math.binghamton.edu
} 
a Poisson subordinator is given in Pickands [15]. Cox processes are also Poisson processes with randomized times. These are also called 'doubly stochastic Poisson processes' (see [16] and [7]). Kumar et al. [9] considered time-changed Poisson processes where the subordinator is an inverse Gaussian process, i.e. the hitting time process of the standard Brownian motion. There are fractional Poisson processes, which are processes with randomized continuous fractional diffusions (see [2], [3], and [11]). There are examples where the random times are Brownian motions (see [4]). For other types of iterated Poisson process; see, e.g. [8].

Let us consider a compound Poisson process (CPP),

$$
Y(t)=\sum_{n=1}^{M(t)} X_{n}, \quad t>0
$$

with $M(t)$ a Poisson process, and $X_{n}$ a sequence of independent and identically distributed (i.i.d.) random variables independent of $M(t)$. In this present paper we investigate the distribution of the process $Z(t)=Y[N(t)]$, where $N(t)$ is an independent Poisson process. Such a process is called a CPP with a Poisson subordinator. We provide explicit equations of this distribution, its moments, and other characteristics. Precisely, after some preliminary results concerning the distribution of the CPP $Y(t)$ given in Section 2, in Section 3 we obtain the probability distribution of the process $Z(t)$ in terms of the Bell polynomials. Then in Sections 4 and 5, we develop the equations of the distribution of $Z(t)$ in the special cases when $Y(t)$ has exponential jumps and normal jumps, respectively.

In order to demonstrate the usefulness of the CPP with a Poisson subordinator we now provide two examples of applications in biomedical research and ecology.

Application 1. Assume that patients having the same disease arrive at a clinic at random times, according to the Poisson process $N(t)$. Each patient receives the same treatment and is under inspection for one time unit (a year). The symptoms of the disease may reoccur at random times during the inspection period, according to the independent Poisson process $M(t)$. The total number of occurrences and their severity is distributed for each patient as the random variable $(R V) Y(1)$. Thus, the process $Z[N(t)]$ represents the total damage inflicted by the disease to the patients after their inspection period.

Application 2. Let $N(t)$ be a Poisson process describing the number of animals caught in $[0, t]$ during an investigation in ecology. A radio transmitter is attached to each animal for a period of one time unit. A transmitter sends information at random, according to an independent Poisson process $M(t)$. The total number of occurrences and the amount of information is distributed for each animal as the $R V Y(1)$. Thus, the process $Z[N(t)]$ represents the total amount of information transmitted by the animals after the investigation period.

The second part of the paper is devoted to a special case, namely the iterated Poisson process, which is a Poisson process whose randomized time is another independent Poisson process. This is actually a CPP whose independent jumps are discrete RVs having a Poisson distribution. An example in queueing theory involving the iterated Poisson process is provided in Application 3. We remark that the probability law, the governing equations, its representation as a random sum, and various generalizations of the iterated Poisson process have been studied in [12] and [13]. For such a process, in Section 6 we express in series form the mean sojourn time in a fixed state. Moreover, we also find conditions under which it converges to a regular Poisson process. Stopping time problems for the iterated Poisson process are finally studied in Section 7. In the case of constant boundaries we obtain the first-crossing time density and 
the mean first-crossing time in closed form. For linear increasing boundaries we develop a computationally effective procedure able to determine iteratively the first-hitting-time density and, in turn, the corresponding survival function.

\section{Preliminaries}

In the present section we bring some well-known results concerning the distribution of the CPP, $Y(t)$, and Bell polynomials, which are used later in the distribution of $Z(t)=Y[N(t)]$.

Consider the CPP $\{Y(t), t \geq 0\}$ defined as

$$
Y(t)=\sum_{n=1}^{M(t)} X_{n}, \quad t>0
$$

where $\{M(t), t \geq 0\}$ is a Poisson process with intensity $\mu$, and $\left\{X_{n}, n \geq 1\right\}$ is a sequence of i.i.d. RVs independent of $\{M(t), t \geq 0\}$. The probability mass function of $M(t)$ at $m$ will be denoted as

$$
p(m ; \mu t)=\mathbb{P}\{M(t)=m\}=\mathrm{e}^{-\mu t} \frac{(\mu t)^{m}}{m !}, \quad m=0,1, \ldots
$$

We assume that $Y(0)=0$ and $M(0)=0$. Let $H_{Y}(y ; t)$ denote the cumulative distribution function (CDF) of $Y(t), y \in \mathbb{R}$ and $t>0$. From (2.1) it follows that if $M(t)=0$ then $Y(t)=0$ so that the distribution of $Y(t)$ has an atom at 0 with $\mathbb{P}\{Y(t)=0\} \geq p(0 ; \mu t)=\mathrm{e}^{-\mu t}, t \geq 0$, where $\mathbb{P}$ denotes the probability measure. Moreover, recalling (2.2) it follows that the CDF of $Y(t)$ is given by the following Poisson mixture:

$$
\begin{aligned}
H_{Y}(y ; t) & =\sum_{m=0}^{+\infty} p(m ; \mu t) F_{X}^{(m)}(y), \\
& =\mathbf{1}_{\{y \geq 0\}} \mathrm{e}^{-\mu t}+\sum_{m=1}^{+\infty} p(m ; \mu t) F_{X}^{(m)}(y), \quad y \in \mathbb{R}, t \geq 0 .
\end{aligned}
$$

Note that in (2.3) and throughout the paper, $g^{(m)}$ denotes the $m$-fold convolution of a given function $g$. The indicator function is denoted by $\mathbf{1}_{\{\cdot\}}$.

If the RVs $X_{i}$ are absolutely continuous with probability density function $f_{X}(\cdot)$ then due to $(2.3)$,

(i) the absolutely continuous component of the probability law of $Y(t)$ is expressed by the density

$$
h_{Y}(y ; t)=\sum_{m=1}^{+\infty} p(m ; \mu t) f_{X}^{(m)}(y), \quad y \neq 0, t>0,
$$

(ii) the discrete component is given by $\mathbb{P}\{Y(t)=0\}=p(0 ; \mu t)=\mathrm{e}^{-\mu t}, t \geq 0$.

Let $M_{X}(s):=\mathbb{E}\left\{\mathrm{e}^{s X}\right\}$ be the moment generating function of $X_{i}$, where $s$ is within the region of convergence of $M_{X}(s)$ and $\mathbb{E}$ is the expectation. From (2.1) it follows that the moment generating function of $Y(t)$ is

$$
\mathbb{E}\left\{\mathrm{e}^{s Y(t)}\right\}=\exp \left\{-\mu t\left[1-M_{X}(s)\right]\right\}, \quad t \geq 0 .
$$


In the following section we study the process $Y(t)$ when the time is randomized according to a Poisson process. Precisely, we consider the stochastic process $\{Y[N(t)], t \geq 0\}$, where $\{N(t), t \geq 0\}$ is a Poisson process with intensity $\lambda$, independent of $Y(t)$. We will express the CDF of $\bar{Z}(t)$ in terms of Bell polynomials

$$
B_{n}(x)=\sum_{k=0}^{\infty} k^{n} \frac{x^{k}}{k !} \mathrm{e}^{-x}, \quad n \geq 0 .
$$

We therefore recall some properties of these polynomials (see, e.g. [5]). Let $B_{n}(x), x \geq 0$, represent the $n$th moment of a Poisson distribution with mean $x$. Obviously, $B_{0}(x)=1$, $B_{1}(x)=x$, and $B_{2}(x)=x+x^{2}$. Generally, $B_{n}(x)$ is a polynomial of degree $n$ of the form

$$
B_{n}(x)=x+a_{2} x^{2}+\cdots+a_{n-1} x^{n-1}+x^{n}, \quad n \geq 2 .
$$

We can determine $B_{n}(x)$ by differentiating the moment generating function $\exp \left\{-x\left(1-\mathrm{e}^{\theta}\right)\right\}$ with respect to $\theta, n$ times, and evaluating the derivative at $\theta=0$. From (2.5) we immediately obtain the derivative

$$
B_{n}^{\prime}(x)=-B_{n}(x)+\frac{B_{n+1}(x)}{x}, \quad n \geq 0 .
$$

Hence, due to (2.6) the following recursive formula holds:

$$
B_{n+1}(x)=x\left[B_{n}^{\prime}(x)+B_{n}(x)\right], \quad n \geq 0,
$$

which is useful in obtaining the explicit form of the Bell polynomials. For instance, according to $(2.7)$, we have

$$
\begin{aligned}
& B_{3}(x)=x+3 x^{2}+x^{3}, \\
& B_{4}(x)=x+7 x^{2}+6 x^{3}+x^{4}, \\
& B_{5}(x)=x+15 x^{2}+25 x^{3}+10 x^{4}+x^{5}
\end{aligned}
$$

etc. Moreover, we can also express these polynomials by the Dobiński formula,

$$
B_{n}(x)=\sum_{k=0}^{n} S_{2}(n, k) x^{k} \quad \text { for } n=0,1, \ldots,
$$

where

$$
S_{2}(n, k)=\left\{\begin{array}{l}
n \\
k
\end{array}\right\}=\frac{1}{k !} \sum_{i=0}^{k}(-1)^{i}\left(\begin{array}{l}
k \\
i
\end{array}\right)(k-i)^{n} \quad \text { for } k=0,1, \ldots, n,
$$

are the Stirling numbers of the second kind.

\section{The distribution of $Z(t)=Y[N(t)]$}

First of all we point out that, due to $(2.1)$, the process $\{Y[N(t)], t \geq 0\}$ is identically distributed to the CPP

$$
Z(t):=\sum_{n=0}^{N(t)} W_{n}, \quad t \geq 0
$$


where $W_{0}=0$ almost surely (a.s.), and $W_{1}, W_{2}, \ldots$ are i.i.d. RVs distributed as

$$
Y(1)=\sum_{n=1}^{M(1)} X_{n}
$$

Note that the CDF of $W_{n}, n \geq 1$, is $H_{Y}(y ; 1)$. The $n$-fold convolution of this CDF is $H_{Y}(y ; n), n \geq 1$. We denote by $H_{Z}(z ; t)$ the CDF of $Z(t)$.

Proposition 3.1. For all $z \in \mathbb{R}$ and $t>0$ the CDF of process (3.1) is

$$
H_{Z}(z ; t)=\exp \left\{-\lambda t\left(1-\mathrm{e}^{-\mu}\right)\right\}\left[\mathbf{1}_{\{z \geq 0\}}+\sum_{n=1}^{+\infty} \frac{\mu^{n}}{n !} B_{n}\left(\lambda t \mathrm{e}^{-\mu}\right) F_{X}^{(n)}(z)\right] .
$$

Proof. From (3.1) and (2.3) we have

$$
\begin{aligned}
H_{Z}(z ; t) & =\sum_{n=0}^{+\infty} p(n ; \lambda t) H_{Y}(z ; n) \\
& =\sum_{n=0}^{+\infty} p(n ; \lambda t)\left[\mathbf{1}_{\{z \geq 0\}} \mathrm{e}^{-\mu n}+\sum_{m=1}^{+\infty} p(m ; \mu n) F_{X}^{(m)}(z)\right], \quad z \in \mathbb{R}, t>0 .
\end{aligned}
$$

In addition,

$$
\sum_{n=0}^{\infty} p(n ; \lambda t) \mathrm{e}^{-\mu n}=\mathrm{e}^{-\lambda t\left(1-\mathrm{e}^{-\mu}\right)}
$$

also, since all terms are nonnegative, and recalling (2.5),

$$
\begin{aligned}
\sum_{n=0}^{\infty} p(n ; \lambda t) \sum_{m=1}^{\infty} p(m ; \mu n) F_{X}^{(m)}(z) & =\sum_{m=1}^{\infty} \frac{\mu^{m}}{m !} F_{X}^{(m)}(z) \sum_{n=0}^{\infty} n^{m} \mathrm{e}^{-\lambda t} \frac{\left(\lambda t \mathrm{e}^{-\mu}\right)^{n}}{n !} \\
& =\mathrm{e}^{-\lambda t\left(1-\mathrm{e}^{-\mu}\right)} \sum_{m=1}^{\infty} \frac{\mu^{m}}{m !} B_{m}\left(\lambda t \mathrm{e}^{-\mu}\right) F_{X}^{(m)}(z) .
\end{aligned}
$$

This proves (3.2).

Corollary 3.1. If $F_{X}$ is absolutely continuous with density $f_{X}$ then the density of (3.2) for any $z \neq 0$ and $t>0$ is

$$
h_{Z}(z ; t)=\exp \left\{-\lambda t\left(1-\mathrm{e}^{-\mu}\right)\right\} \sum_{n=1}^{+\infty} \frac{\mu^{n}}{n !} B_{n}\left(\lambda t \mathrm{e}^{-\mu}\right) f_{X}^{(n)}(z) .
$$

Remark 3.1. Note that if $F_{X}$ is continuous at 0 then $H_{Z}(z ; t)$ has a jump of size $\mathrm{e}^{-\lambda t\left(1-\mathrm{e}^{-\mu}\right)}$ at $z=0$.

Remark 3.2. We can easily prove that $H_{Z}(z ; t)$ given by (3.2) is indeed a CDF.

Recalling that $W_{i}$ is distributed as $Y(1)$, by setting $t=1$ in (2.4) we obtain the following moment generating function:

$$
M_{W}(s):=\mathbb{E}\left\{\mathrm{e}^{s W_{i}}\right\}=\exp \left\{-\mu\left[1-M_{X}(s)\right]\right\}, \quad i=1,2, \ldots
$$


Hence, due to (3.3) the Laplace-Stieltjes transform of $Z(t)$ is

$$
\mathbb{E}\left\{\mathrm{e}^{-\theta Z(t)}\right\}=\exp \left\{-\lambda t\left[1-M_{W}(-\theta)\right]\right\}=\exp \{-t \Psi(\theta)\}, \quad t>0,
$$

where

$$
\Psi(\theta)=\lambda\left[1-\mathrm{e}^{-\mu\left[1-M_{X}(-\theta)\right]}\right]
$$

We recall that $\{Z(t), t \geq 0\}$ is a CPP time-changed by a Poisson process, and, thus, it is a Lévy process (see, e.g. [1, Theorem 1.3.25]).

Let us now focus on the mean and the variance of $Z(t)$. From (3.1) we have

$$
\mathbb{E}\{Z(t)\}=\lambda \mu \xi t, \quad \operatorname{var}[Z(t)]=\lambda \mu\left[\sigma^{2}+(\mu+1) \xi^{2}\right] t, \quad t \geq 0,
$$

where we have set

$$
\xi=\mathbb{E}\left\{X_{1}\right\} \quad \text { and } \quad \sigma^{2}=\operatorname{var}\left(X_{1}\right) .
$$

Finally, by the strong law of large numbers, the following asymptotic result holds for process $Z(t)$ :

$$
\lim _{t \rightarrow+\infty} \frac{Z(t)}{t}=\lambda \mu \xi \quad \text { a.s. }
$$

In the following sections we consider three special cases of interest, in which the distributions of the jump variables $X_{i}$ are assumed to be deterministic, exponential, and normal.

\section{The compound Poisson process with exponential jumps}

In this section we consider the CPP (2.1) when $\left\{X_{n}, n \geq 1\right\}$ are i.i.d. exponentially distributed RVs with parameter $\zeta$, i.e. $f_{X}(x)=\zeta \mathrm{e}^{-\zeta x} \mathbf{1}_{\{x \geq 0\}}$. In this case, we have for $x \geq 0$,

$$
F_{X}(x)=1-p(0 ; \zeta x), \quad F_{X}^{(m)}(x)=1-P(m-1 ; \zeta x), \quad m=1,2 \ldots,
$$

where $p(n ; \lambda)$ is defined in $(2.2)$ and $P(n ; \lambda)$ is the CDF given by

$$
P(n ; \lambda)=\sum_{i=0}^{n} p(i ; \lambda)
$$

Hence, due to (2.3) the CDF of $Y(t), t \geq 0$, is

$$
H_{Y}(y ; t)=\mathrm{e}^{-\mu t}+\sum_{m=1}^{+\infty} p(m ; \mu t)[1-P(m-1 ; \zeta y)], \quad y \geq 0,
$$

Thus, the atom of such a function is $H_{Y}(0 ; t)=\mathrm{e}^{-\mu t}$. Generally, for $n=1,2, \ldots$,

$$
H_{Y}^{(n)}(y ; 1)=H_{Y}(y ; n)=1-\sum_{m=1}^{+\infty} p(m ; n \mu) P(m-1 ; \zeta y), \quad y \geq 0
$$


(a)

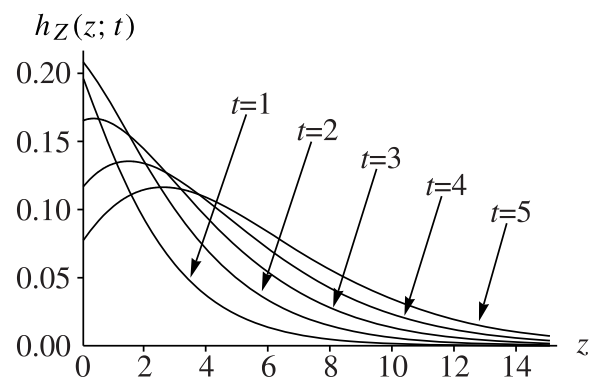

(b)

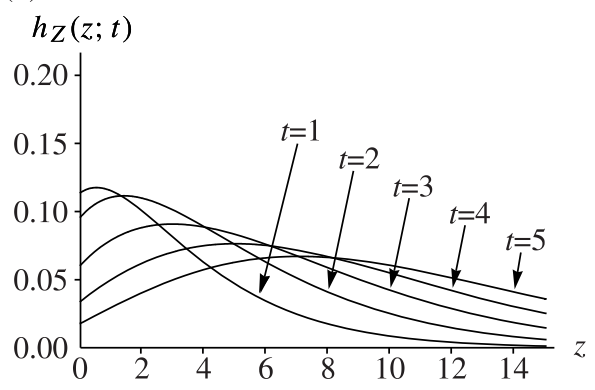

FiguRE 1: Density (4.2) for $\mu=1, \zeta=1$, and various choices of $t$ with (a) $\lambda=1$, and (b) $\lambda=2$. The corresponding probability mass of $h_{Z}(z ; t)$ is $0.4685,0.7175,0.8499,0.9202,0.9576$ in (a), and 0.7175 , $0.9202,0.9775,0.9936,0.9982$ in (b) for increasing values of $t$.

According to Proposition 3.1, the $\mathrm{CDF}$ of $Z(t)$ can be expressed as

$$
\begin{aligned}
H_{Z}(z ; t) & =\sum_{n=0}^{+\infty} p(n ; \lambda t)\left[1-\sum_{m=1}^{+\infty} p(m ; n \mu) P(m-1 ; \zeta z)\right] \\
& =1-\sum_{n=0}^{+\infty} p(n ; \lambda t) \sum_{m=1}^{+\infty} p(m ; n \mu) P(m-1 ; \zeta z) \\
& =1-\sum_{m=1}^{+\infty} p_{m}(t) P(m-1 ; \zeta z),
\end{aligned}
$$

since $p_{m}(t)=\sum_{n=0}^{+\infty} p(n ; \lambda t) p(m ; n \mu)$ due to (6.1). Additional manipulations yield the alternative equation

$$
H_{Z}(z ; t)=\sum_{j=0}^{+\infty} p(j ; \zeta z) \sum_{m=0}^{j} p_{m}(t)
$$

We pinpoint that the atom at 0 of $(4.1)$ is $H_{Z}(0 ; t)=p_{0}(t)$. Since $(\mathrm{d} / \mathrm{d} z) P(m-1 ; \zeta z)=$ $-\zeta p(m-1 ; \zeta z)$, the density of $H_{Z}(z ; t)$ for $z>0$ and $t>0$, is expressed as

$$
h_{Z}(z ; t)=\zeta \sum_{m=1}^{+\infty} p_{m}(t) p(m-1 ; \zeta z)
$$

Some plots of such density are presented in Figure 1, where the probability mass $\int_{0}^{+\infty} h_{Z}(z ; t) \mathrm{d} z$ is shown in the caption for some choices of $t$.

Finally, we note that the expected value of $Z(t)$ is $\mathbb{E}\{Z(t)\}=\lambda \mu t / \zeta$.

\section{The compound Poisson process with normal jumps}

Let us now investigate the CPP (2.1) when $\left\{X_{n}, n \geq 1\right\}$ are i.i.d. normally distributed RVs with mean $\eta \in \mathbb{R}$ and variance $\sigma^{2}>0$, so

$$
F_{X}(x)=\Phi\left(\frac{x-\eta}{\sigma}\right), \quad F_{X}^{(n)}(x)=\Phi\left(\frac{x-n \eta}{\sigma \sqrt{n}}\right), \quad x \in \mathbb{R},
$$


(a)

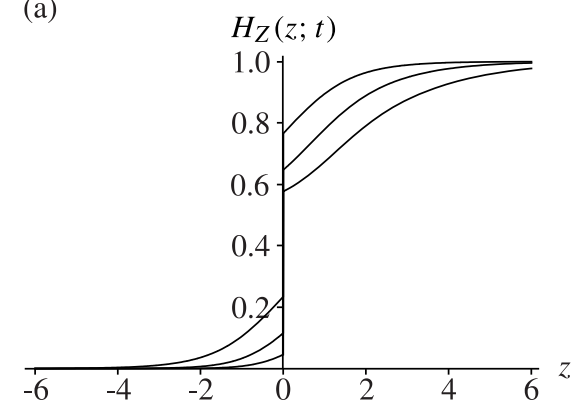

(b)

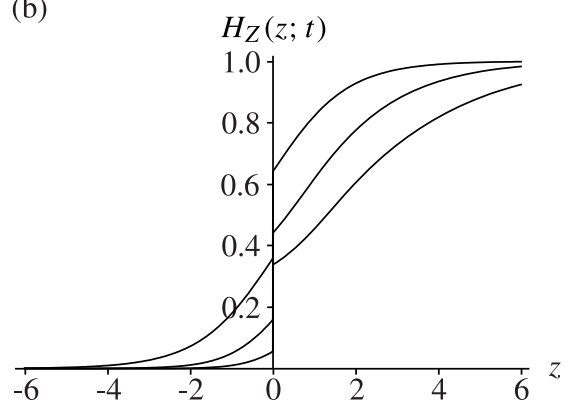

FIGURE 2: Distribution function (5.1) for $\lambda=1, \mu=1, \sigma=1$, and $\eta=0,0.5,1$ (from top to bottom) with (a) $t=1$, and (b) $t=2$.

where $\Phi(\cdot)$ is the standard normal distribution function. Hence, for all $y \in \mathbb{R}$,

$$
H_{Y}^{(n)}(y ; 1)=H_{Y}(y ; n)=\mathrm{e}^{-n \mu} \mathbf{1}_{\{y \geq 0\}}+\sum_{m=1}^{+\infty} p(m ; n \mu) \Phi\left(\frac{y-m \eta}{\sigma \sqrt{m}}\right), \quad t \geq 0 .
$$

Note that the above distribution has support on $(-\infty,+\infty)$, and it is absolutely continuous on all $y \neq 0$ with a jump (atom) at $y=0$. According to (3.2), the $\operatorname{CDF}$ of $Z(t)$ is

$$
H_{Z}(z ; t)=p_{0}(t) \mathbf{1}_{\{z \geq 0\}}+\sum_{n=1}^{+\infty} p_{n}(t) \Phi\left(\frac{z-n \eta}{\sigma \sqrt{n}}\right), \quad z \in \mathbb{R}, t \geq 0,
$$

where $p_{n}(t)$ is given in (6.1). In Figure 2 we show some plots of $H_{Z}(z ; t)$. In this case the Lévy exponent of $\{Z(t), t \geq 0\}$ is

$$
\Psi(\theta)=\lambda\left[1-\exp \left\{-\mu\left(1-\mathrm{e}^{-\eta \theta+\sigma^{2} \theta^{2} / 2}\right)\right\}\right] .
$$

In conclusion, from (5.1) it follows that the density of $Z(t)$, at $z \neq 0$ is

$$
h_{Z}(z ; t)=\frac{1}{\sigma} \sum_{n=1}^{+\infty} p_{n}(t) \frac{1}{\sqrt{n}} \phi\left(\frac{z-n \eta}{\sigma \sqrt{n}}\right)
$$

where $\phi(\cdot)$ is the standard normal density.

\section{The iterated Poisson process}

In this section we suppose that in (3.1) the RVs $W_{n}, n=1,2, \ldots$, have a Poisson distribution with parameter $\mu$. This special case corresponds to the assumption that $X_{i}=1, i \geq 1$ a.s. so that

$$
F_{X}(x)=\mathbf{1}_{\{x \geq 1\}}, \quad F_{X}^{(n)}(x)=\mathbf{1}_{\{x \geq n\}}, \quad n=1,2, \ldots,
$$

and, thus, $Y(t)=M(t), t \geq 0$, a.s. Hence, in this case $\{Z(t), t \geq 0\}$ is a Markovian jump process over the set of nonnegative integers with right-continuous nondecreasing sample paths. This is called an 'iterated Poisson process' with parameters $(\mu, \lambda)$, since it can be expressed as a Poisson process $M(t)$ with intensity $\mu$ whose randomized time is an independent Poisson 
(a)

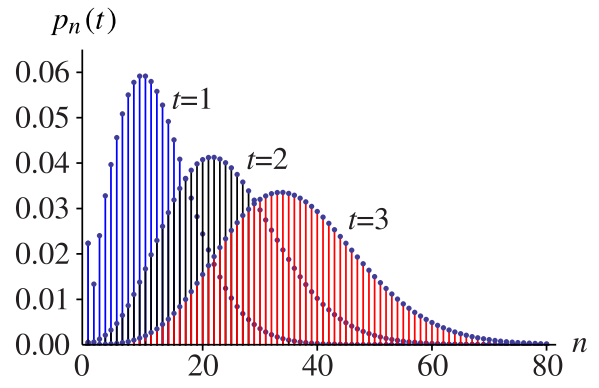

(b)

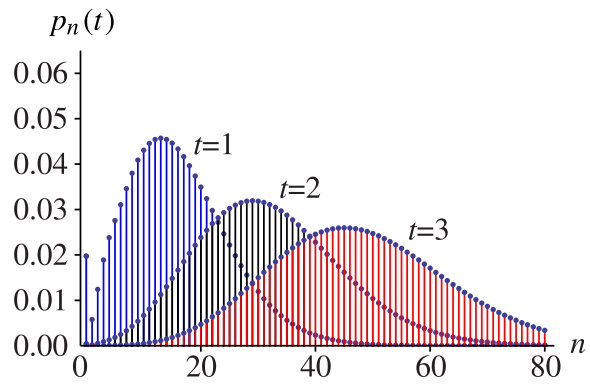

Figure 3: Probability distribution (6.1) for $\lambda=4$ and for (a) $\mu=3$ and (b) $\mu=4$.

process $N(t)$ with intensity $\lambda$, i.e. $Z(t)=M[N(t)]$. We point out that various results on this process have been obtained in [12] and [13].

In analogy with (3.3), the probability function of the iterated Poisson process for $t>0$ is (cf. [12, Equation (8)])

$$
p_{n}(t)=\mathbb{P}\{Z(t)=n\}=\exp \left\{-\lambda t\left(1-\mathrm{e}^{-\mu}\right)\right\} \frac{\mu^{n}}{n !} B_{n}\left(\lambda t \mathrm{e}^{-\mu}\right), \quad n=0,1, \ldots
$$

Note that $\sum_{n=0}^{+\infty} p_{n}(t)=1$ for all $t>0$. We recall that $B_{0}(x)=1$, and that $B_{n}(x)$ can be expressed in closed form by means of the Dobiński formula (2.8). Some plots of $p_{n}(t)$ are shown in Figure 3 for $t=1,2,3$.

As a special case of (3.2) we have the discrete CDF of $Z(t), t \geq 0$ :

$$
P_{n}(t)=H_{Z}(n ; t)=\exp \left\{-\lambda t\left(1-\mathrm{e}^{-\mu}\right)\right\} \sum_{j=0}^{n} \frac{\mu^{j}}{j !} B_{j}\left(\lambda t \mathrm{e}^{-\mu}\right), \quad n=0,1, \ldots
$$

Making use of (6.1) and (2.8) the following alternative form of $P_{n}(t), t \geq 0$, can be obtained:

$$
P_{n}(t)=\exp \left\{-\lambda\left(1-\mathrm{e}^{-\mu}\right) t\right\}\left[1+\mathbf{1}_{\{n \geq 1\}} \sum_{k=0}^{n}\left(\lambda \mathrm{e}^{-\mu} t\right)^{k} \sum_{j=k}^{n} S_{2}(j, k) \frac{\mu^{j}}{j !}\right], \quad n=0,1, \ldots
$$

The Lévy exponent of the iterated Poisson process is

$$
\Psi(\theta)=\lambda\left[1-\exp \left\{-\mu\left(1-\mathrm{e}^{-\theta}\right)\right] .\right.
$$

Note that if $\mu \rightarrow 0$ and $\lambda \rightarrow+\infty$ such that $\lambda \mu \rightarrow \xi$ with $0<\xi<+\infty$ then the right-hand side of (6.4) tends to $\xi\left(1-\mathrm{e}^{-\theta}\right)$, which is the Lévy exponent of the Poisson process with intensity $\xi$.

Remark 6.1. (i) Due to the following well-known recurrence formula of Bell polynomials,

$$
B_{n}(x)=x \sum_{k=1}^{n}\left(\begin{array}{l}
n-1 \\
k-1
\end{array}\right) B_{k-1}(x), \quad n=1,2, \ldots,
$$

the probability distribution of the iterated Poisson process satisfies the recurrence relation

$$
p_{n}(t)=\frac{1}{n} \lambda \mathrm{e}^{-\mu} t \sum_{k=1}^{n} \frac{\mu^{n-k+1}}{(n-k) !} p_{k-1}(t), \quad n=1,2, \ldots, t>0 .
$$


(ii) The following conditional probability holds for $0<s<t$ and $k=0,1, \ldots, n$ :

$$
\mathbb{P}\{Z(s)=k \mid Z(t)=n\}=\left(\begin{array}{l}
n \\
k
\end{array}\right) \frac{B_{k}\left(\lambda \mathrm{e}^{-\mu} s\right) B_{n-k}\left(\lambda \mathrm{e}^{-\mu}(t-s)\right)}{B_{n}\left(\lambda \mathrm{e}^{-\mu} t\right)} .
$$

If $t \rightarrow+\infty$ and $s \rightarrow+\infty$ with $s / t \rightarrow \theta \in(0,1)$ then the right-hand side of (6.6) tends to a binomial distributions with parameters $n$ and $\theta$, since $B_{n}(x) \sim x^{n}$ for $x$ large.

Recalling (see [13]) that the mean and the variance of $Z(t)$ are given by $\mathbb{E}\{Z(t)\}=\lambda \mu t$ and $\operatorname{var}[Z(t)]=\lambda \mu(1+\mu) t, t \geq 0$, respectively, it follows that the iterated Poisson process is overdispersed, since its dispersion index

$$
\frac{\operatorname{var}[Z(t)]}{\mathbb{E}\{Z(t)\}}=1+\mu
$$

is larger than 1 and, in particular, is independent of time $t$.

Finally, let us denote by $S_{n}$ the sojourn time of the iterated Poisson process $Z(t)$ in state $n$. Since $Z(0)=0$ a.s. and

$$
\lim _{h \rightarrow 0^{+}} \frac{1}{h} \mathbb{P}\{Z(t+h) \neq k \mid Z(t)=k\}=\lambda\left(1-\mathrm{e}^{-\mu}\right), \quad k=0,1, \ldots
$$

then $S_{0}$ is exponentially distributed with parameter $\lambda\left(1-\mathrm{e}^{-\mu}\right)$. Moreover, $Z(t)$ is a transient Markov process over the nonnegative integers, so its mean sojourn time in state $n$ is finite. Indeed, due to (6.1),

$$
\mathbb{E}\left\{S_{n}\right\}=\int_{0}^{+\infty} p_{n}(t) \mathrm{d} t=\frac{1}{\lambda} \frac{\mu^{n}}{n !} \sum_{k=1}^{+\infty} k^{n} \mathrm{e}^{-\mu k}<+\infty .
$$

We conclude this section with an example of an application of the iterated Poisson process to queueing theory, which also highlights a context in which the results given in the next section are relevant.

Application 3. Consider a M/D/1 queueing system having arrival rate $\lambda$ and constant unity service time. Assume that the service station is inactive during the time interval $[0, t]$ so that all waiting customers arriving in this period wait in a queueing room. At time the bulk service of all customers begins, and, thus, ends after a time period whose length is equal to the number of customers arrived in $[0, t]$. Assume that during such service time other customers join the system with rate $\mu$. Thus, the iterated Poisson process $Z(t)$ describes the number of customers that join the system during the service period of those arrived in $[0, t]$. In this context, the upward first-crossing time through a constant boundary is the first time that the number of customers $Z(t)$ crosses a fixed threshold, such as a waiting-room capacity.

\section{Stopping time problems for an iterated Poisson process}

Stimulated by the potential applications, in this section we study some stopping time problems for the iterated Poisson process.

The problem of determining the first-passage time distribution for time-changed Poisson processes through a constant boundary has been considered recently in [14] with detailed attention given to the cases of the fractional Poisson process, the relativistic Poisson process, 
(a)

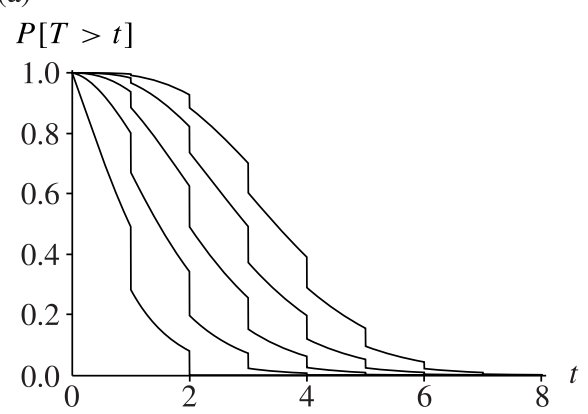

(b)

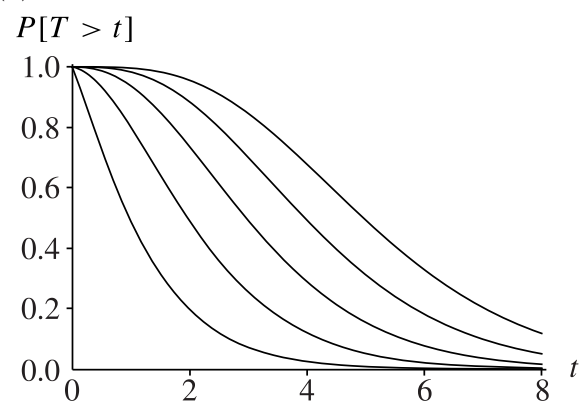

FIGURE 4: Plots of (7.2) for $k=2,4,6,8$, and 10 (from bottom to top) with $\mu=1, \lambda=2$, and boundary (a) $\beta_{k}(t)=k-t$, and (b) $\beta_{k}(t)=k$.

and the gamma Poisson process. Moreover, some results on the distributions of stopping times of CPPs in the presence of linear boundaries are given in [6], [17], [18], and [19].

In this section we confront the first-crossing time problem through various types of boundary for the iterated Poisson process $Z(t)$ investigated in the previous section.

Let $\beta_{k}(t)$ be a continuous function such that $\beta_{k}(t) \geq 0$ for all $t \geq 0$, and $\beta_{k}(0)=k$ with $k \in \mathbb{N}^{+}$. The first-crossing time of $Z(t)$, defined as in (3.1), through the boundary $\beta_{k}(t)$ will be denoted as

$$
T=\inf \left\{t>0: Z(t) \geq \beta_{k}(t)\right\} .
$$

Since $Z(0)=0<\beta_{k}(0)$, the first crossing occurs from below.

Proposition 7.1. Let $Z(t)$ be the iterated Poisson process. If $\beta_{k}(t)$ is nonincreasing in $t$ then for all $t \geq 0$,

$$
\mathbb{P}\{T>t\}=P_{\left\lfloor\beta_{k}(t)^{-}\right\rfloor}(t),
$$

where $\left\lfloor x^{-}\right\rfloor$is the largest integer smaller than $x$, and $P_{n}(t)$ has been given in (6.3).

Proof. The proof follows immediately, since the sample paths of $Z(t)$ are nondecreasing and, thus, $\mathbb{P}\{T>t\}=\mathbb{P}\left\{Z(t)<\beta_{k}(t)\right\}$ for all $t \geq 0$.

Some plots of the survival function of $T$ are shown in Figure 4 for a linear decreasing boundary and for a constant boundary.

\subsection{Constant boundary}

We now obtain the probability law of (7.1) when the boundary is constant. In this case the first-crossing time density of $T$ will be denoted by $\psi(t)$.

Proposition 7.2. Let $Z(t)$ be the iterated Poisson process. If $\beta_{k}(t)=k$ for all $t \geq 0$ then for $k=1$ the first-crossing time density is exponential with parameter $\lambda\left(1-\mathrm{e}^{-\mu}\right)$, whereas for $k=2,3, \ldots$,

$$
\begin{aligned}
\psi(t)= & p_{0}(t) \lambda\left(1-\mathrm{e}^{-\mu}\right)\left[1+\sum_{i=0}^{k-1}\left(\lambda \mathrm{e}^{-\mu} t\right)^{i} C(i ; k-1)\right] \\
& -p_{0}(t) \lambda \mathrm{e}^{-\mu} \sum_{i=1}^{k-1} i\left(\lambda \mathrm{e}^{-\mu} t\right)^{i-1} C(i ; k-1)
\end{aligned}
$$


where

$$
C(i ; k-1):=\sum_{j=i}^{k-1} S_{2}(j, i) \frac{\mu^{j}}{j !} .
$$

Proof. From Proposition 7.1, which includes the case of constant boundaries, we have

$$
\mathbb{P}\{T>t\}=P_{k-1}(t) \quad \text { for all } t \geq 0 .
$$

Due to (7.4) the first-crossing time density through state $k$ can be expressed as $\psi(t)=$ $-(\mathrm{d} / \mathrm{d} t) \mathbb{P}\{T>t\}=-(\mathrm{d} / \mathrm{d} t) P_{k-1}(t)$. Hence, differentiating (6.3) with respect to $t$ yields (7.3).

We remark that under the assumptions of Proposition 7.2, due to (6.2) and (7.4), $T$ is an honest RV. Let us then evaluate the mean of $T$.

Corollary 7.1. Let $Z(t)$ be the iterated Poisson process. If $\beta_{k}(t)=k$ for all $t \geq 0$ then

$$
\mathbb{E}\{T\}=\frac{1}{\lambda\left(1-\mathrm{e}^{-\mu}\right)}\left[1+\mathbf{1}_{\{n \geq 1\}} \sum_{k=0}^{n} \frac{k !}{\left(\mathrm{e}^{\mu}-1\right)^{k}} \sum_{j=k}^{n} S_{2}(j, k) \frac{\mu^{j}}{j !}\right], \quad k \in \mathbb{N}^{+} .
$$

Proof. Since $\mathbb{E}\{T\}=\int_{0}^{+\infty} \mathbb{P}\{T>t\} \mathrm{d} t$, making use of (6.3) and (7.4) after some calculations we obtain (7.5).

Recall that the iterated Poisson process $Z(t)$ is a Markov process over the nonnegative integers. We denote by

$$
H=\inf \{t>0: Z(t)=k\}, \quad k \in \mathbb{N}^{+},
$$

the first-hitting time of state $k$ for $Z(t)$. Let $h(t)$ denote the density of $H$.

Proposition 7.3. Let $Z(t)$ be the iterated Poisson process. The density of the first-hitting time $H$ for $t>0$ is given by

$$
h(t)=\frac{\mathrm{e}^{-\mu} \mu^{k}}{k !} \lambda \exp \left\{-\lambda\left(1-\mathrm{e}^{-\mu}\right) t\right\} B_{k}^{\prime}\left(\lambda \mathrm{e}^{-\mu} t\right), \quad k=1,2, \ldots,
$$

where $B_{k}^{\prime}(x)$ is defined in (2.6).

Proof. For any $t>0$ and $k=1,2, \ldots$, we have

$$
h(t) \mathrm{d} t=\sum_{j=0}^{k-1} \mathbb{P}\{Z(t)=j\} \mathbb{P}\{Z(\mathrm{~d} t)=k-j\} .
$$

Making use of (6.1) and by setting $i=j+1$ we obtain

$$
h(t)=\frac{\mathrm{e}^{-\mu} \mu^{k}}{k !} \lambda \exp \left\{-\lambda\left(1-\mathrm{e}^{-\mu}\right) t\right\} \sum_{i=1}^{k}\left(\begin{array}{c}
k \\
i-1
\end{array}\right) B_{i-1}\left(\lambda \mathrm{e}^{-\mu} t\right) .
$$

The density (7.6) then follows by recalling the recurrence equation (6.5).

Some plots of the first-hitting time density (7.6) are shown in Figure 5.

In the following proposition we evaluate the distribution function of the first-hitting time, and express it in terms of the lower incomplete gamma function $\gamma(a, z)=\int_{0}^{z} t^{a-1} \mathrm{e}^{-t} \mathrm{~d} t$. 
(a)

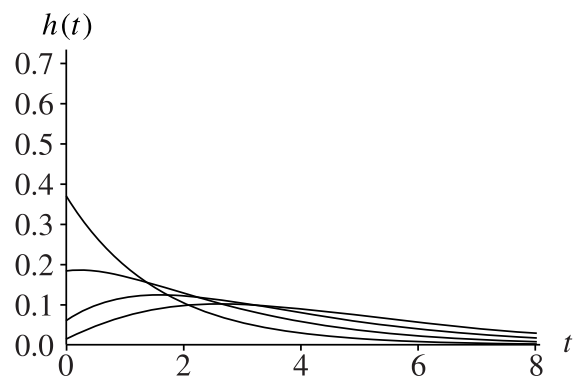

(b)

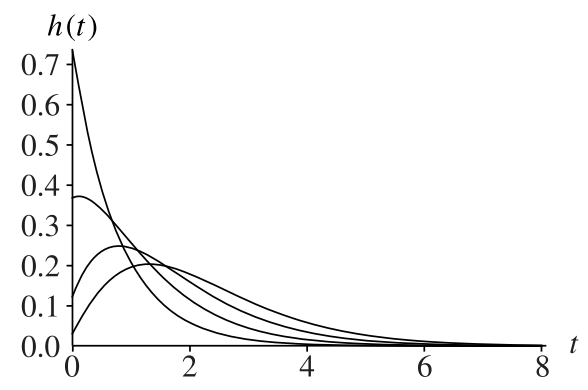

FIGURE 5: First-hitting time density (7.6) with $\mu=1$ and (a) $\lambda=1$ and (b) $\lambda=2$ for $k=1,2,3,4$ (from top to bottom near the origin).

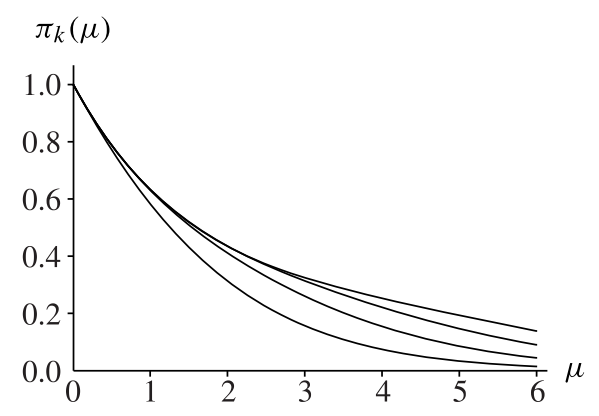

FIGURE 6: First-hitting probability (7.8) as a function of $\mu$ for $k=1,2,3,4$ (from bottom to top).

Proposition 7.4. Let $Z(t)$ be the iterated Poisson process. The distribution function of $H$ for $k=1,2, \ldots$ and $t \geq 0$ is given by

$$
F_{H}(t)=\frac{\mu^{k}}{k !}\left[\exp \left\{-\lambda\left(1-\mathrm{e}^{-\mu}\right) t\right\} B_{k}\left(\lambda \mathrm{e}^{-\mu} t\right)+\sum_{j=0}^{k} S_{2}(k, j) \frac{\gamma\left(j+1, \lambda\left(1-\mathrm{e}^{-\mu}\right) t\right)}{\left(\mathrm{e}^{\mu}-1\right)^{j}}\right] .
$$

Proof. Making use of (7.6) and setting $\lambda \mathrm{e}^{\mu} \tau=x$ we have

$$
F_{H}(t)=\int_{0}^{t} h(\tau) \mathrm{d} \tau=\frac{\mu^{k}}{k !} \int_{0}^{\lambda \mathrm{e}^{-\mu} t} \exp \left\{-\left(\mathrm{e}^{\mu}-1\right) x\right\} B_{k}^{\prime}(x) \mathrm{d} x .
$$

Integrating by parts and recalling the Dobiński formula (2.8) after some calculations, we finally obtain (7.7).

From (7.7) it is not hard to see that the first-hitting probability is

$$
\pi_{k}:=\mathbb{P}\{H<\infty\}=\frac{\mu^{k}}{k !} \sum_{j=1}^{k} S_{2}(k, j) \frac{j !}{\left(\mathrm{e}^{\mu}-1\right)^{j}}, \quad k=1,2, \ldots
$$

It is interesting to note that this probability does not depend on $\lambda$. Plots of $\pi_{k}=\pi_{k}(\mu)$ are shown in Figure 6 for some choices of $k$. Note that the first-hitting probability (7.8) is in general less than unity. 


\subsection{Linear increasing boundary}

Let us now consider the first-crossing time $T$ of $Z(t)$ through a linear boundary with unity slope $\beta_{k}(t)=k+t$, where $k$ is a fixed nonnegative integer. For all nonnegative integers $j$ we define the avoiding probability

$$
g(j ; t)=\mathbb{P}\{Z(t)=j, T>t\}, \quad t \geq 0 .
$$

Clearly, we have $g(j ; t)=0$ for all $j \geq k+t$. Moreover, for all $t \geq 0$ we have

$$
g(j ; t)=p_{j}(t) \text { for } 0 \leq j \leq k .
$$

The case $j>k$ will be investigated below.

We first give a recursive procedure able to evaluate probabilities (7.9) for integer values of $t$.

Proposition 7.5. Let $Z(t)$ be the iterated Poisson process, and let $\beta_{k}(t)=k+t$. The following steps provide an iterative procedure to evaluate $g(j ; n)$ with $n$ integer:

- for $n=0, g(0 ; 0)=1$;

- for $n=1, g(j ; 1)=p_{j}(1)$ for $0 \leq j \leq k$;

- for $n=2,3, \ldots$,

$$
g(j ; n)= \begin{cases}\sum_{i=0}^{j} g(i ; n-1) p_{j-i}(1), & 0 \leq j \leq k+n-2, \\ \sum_{i=0}^{k+n-2} g(i ; n-1) p_{k+n-1-i}(1), & j=k+n-1 .\end{cases}
$$

Proof. We recall that $p_{n}(t)=\sum_{j=0}^{n} p_{j}(s) p_{n-j}(t-s)$ for $0<s<t$. Hence, the proof follows by the definitions of the involved quantities and the properties of $Z(t)$.

Let us now determine the survival function of $T$ under the assumptions of Proposition 7.5.

Proposition 7.6. Let $Z(t)$ be the iterated Poisson process, and let $\beta_{k}(t)=k+t$. For any $t>0$ the survival function of $T$ is given by

$$
\mathbb{P}\{T>t\}= \begin{cases}\sum_{\substack{i=0 \\ k+n}}^{k+n-1} g(i ; n), & t=n, \\ \sum_{i=0}^{k+n} g(i ; t)=\sum_{i=0}^{k+n} \sum_{m=0}^{i} g(m ; n) p_{i-m}(t-n), & n<t<n+1,\end{cases}
$$

where $n$ denotes a nonnegative integer, and in the last sum we set $g(k+n ; n) \equiv 0$.

Proof. From (7.9) we have

$$
\mathbb{P}\{T>t\}=\sum_{j<k+t} g(j ; t) .
$$

Hence, the proof follows from Proposition 7.5 and the Markov property of $Z(t)$.

In conclusion, in Figure 7 we show some plots of the survival function of $T$ obtained by use of Proposition 7.6. 


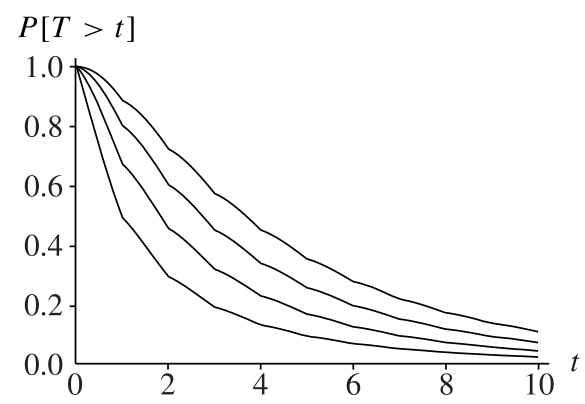

Figure 7: Plot of (7.10) for $\mu=1$ and $\lambda=2$ with $k=1,2,3,4$ (from bottom to top), when $\beta_{k}(t)=k+t$.

\section{Acknowledgements}

The research of A. Di Crescenzo and B. Martinucci has been performed under partial support by Regione Campania (Legge 5) and GNCS-INdAM. S. Zacks is grateful to the Ph.D. Program in Mathematics of Salerno University for his partial support during a visit in July 2010.

\section{References}

[1] Applebaum, D. (2009). Lévy Processes and Stochastic Calculus, 2nd edn. Cambridge University Press.

[2] Beghin, L. ANd Orsingher, E. (2009). Fractional Poisson processes and related planar random motions. Electron. J. Prob. 14, 1790-1827.

[3] Beghin, L. And Orsingher, E. (2010). Poisson-type processes governed by fractional and higher-order recursive differential equations. Electron. J. Prob. 15, 684-709.

[4] Beghin, L. And Orsingher, E. (2012). Poisson process with different Brownian clocks. Stochastics 84, 79-112.

[5] Сомtet, L. (1974). Advanced Combinatorics. The Art of Finite and Infinite Expansions. Reidel, Dordrecht.

[6] Di Crescenzo, A. and Martinucci, B. (2009). On a first-passage-time problem for the compound power-law process. Stoch. Models 25, 420-435.

[7] Grandell, J. (1976). Doubly Stochastic Poisson Processes (Lecture Notes Math. 529). Springer, Berlin.

[8] Horváth, L. and Steinebach, J. (1999). On the best approximation for bootstrapped empirical processes. Statist. Prob. Lett. 41, 117-122.

[9] Kumar, A., Nane, E. and Vellaisamy, P. (2011). Time-changed Poisson processes. Statist. Prob. Lett. 81, 1899-1910.

[10] Lee, M. L. T. and Whitmore, G. A. (1993). Stochastic processes directed by randomized time. J. Appl. Prob. 30, 302-314.

[11] Mainardi, F., Gorenflo, F. and Scalas, E. (2004). A fractional generalization of the Poisson processes. Vietnam J. Math. 32, 53-64.

[12] Orsingher, E. And Polito, F. (2010). Composition of Poisson processes. In Proceedings of XIV International Conference on Eventological Mathematics and Related Fields (Krasnoyarsk, Russia), pp. 13-18.

[13] Orsingher, E. ANd Polito, F. (2012). Compositions, random sums and continued random fractions of Poisson and fractional Poisson processes. J. Statist. Phys. 148, 233-249.

[14] Orsingher, E. And Toaldo, B. (2015). Counting processes with Bernštein intertimes and random jumps. To appear in J. Appl. Prob. $\mathbf{5 2}$.

[15] Pickands, J., III (1971). The two-dimensional Poisson process and extremal processes. J. Appl. Prob. 8, $745-756$.

[16] Serfozo, R. F. (1972). Conditional Poisson processes. J. Appl. Prob. 9, 288-302.

[17] Stadje, W. and Zacks, S. (2003). Upper first-exit times of compound Poisson processes revisited. Prob. Eng. Inf. Sci. 17, 459-465.

[18] ZACKs, S. (1991). Distributions of stopping times for Poisson processes with linear boundaries. Commun. Statist. Stoch. Models 7, 233-242.

[19] ZACKS, S. (2005). Some recent results on the distributions of stopping times of compound Poisson processes with linear boundaries. J. Statist. Planning Infer. 130, 95-109. 